\title{
Wide Field Photometric Study of Galactic Cluster NGC 6067
}

\author{
Alok K. Durgapal ${ }^{1}$, Geeta Rangwal ${ }^{1}$ and R. K. S. Yadav ${ }^{2}$ \\ ${ }^{1}$ Department of Physics (UGC-Centre of Advanced Study), Kumaun University, Nainital, India. \\ ${ }^{2}$ Aryabhatta Research Institute of Observational Sciences, Manora Peak, Nainital 263002, India. \\ Email: alokdurgapal@gmail.com
}

\begin{abstract}
We conduct wide field BV photometry of open cluster NGC 6067 using the archive data taken from WFI@2.2 catalogue. The astrophysical parameters of the cluster such as age, cluster center and heliocentric distance using colour magnitude diagrams are calculated. We also calculated the value of interstellar extinction in the direction of this cluster.
\end{abstract}

Keywords: Wide field photometry, Galactic clusters, Colour magnitude diagrams, Cluster parameters.

\section{Introduction}

Open star clusters are groups of gravitationally bound stars and are almost at same distance from us. Open star clusters span a large age range (Durgapal and Pandey, 2001), so are good laboratories to study the star evolution theories. NGC 6067 is very poorly studied young open star cluster. Thackeray et al. (1962) studied this cluster using photographic and photoelecrtic data. They estimated radial velocity and parallaxes for the cluster.

In this analysis we performed BV CCD photometric study of the open star cluster NGC 6067. In next section, we have discussed the data used and its reduction techniques. After this we described the present analysis and methods which are used to determine the basic parameters of the cluster NGC 6067. In last section we concluded our analysis and present the main results of this study.

\section{Data}

We used optical CCD archival data of WFI@2.21 for the cluster NGC 6067, which was observed with Wide Field Imager (WFI) at $2.2 \mathrm{~m}$ ESO/MPI telescope La Silla, Chile. We used total 48 images in $\mathrm{B}$ and $\mathrm{V}$ band for this analysis. The images were reduced using procedures discussed by Anderson et al. (2006). Data from WEBDA database are used to calibrate the instrumental magnitudes.

\section{Astrophysical parameters}

The cluster center have been estimated using star count method. For this purpose we have divided the cluster region in small bins. A histogram of right ascension (RA) and declination (DEC) against the stars in each bin was plotted and shown in Fig. 1. The central density was determined by fitting Gaussian function over these histograms, which is denoted by a dashed line in Fig. 1. The central coordinates were estimated as $243.304 \pm 0.01$ deg and $-54.225 \pm 0.004 \mathrm{deg}$ in RA and DEC respectively. The astrophysical parameters such as age, metal content, distance modulus and reddening are determined by isochrone fitting method. We plotted the V versus (B-V) colourmagnitude diagram (CMD) for the cluster stars. 
The CMD for the cluster is shown in Fig. 2. We fitted the isochrones of Marigo et al. (2017) on the cluster CMD. These includes thermally pulsing asymptotic giant branch phase. The best fit was found with the isochrone of age $129 \mathrm{Myr}$ and metallicity $\mathrm{Z}=0.01$.

\section{Results and conclusions}

In this analysis with the help of wide field BV CCD photometry for the open star cluster NGC

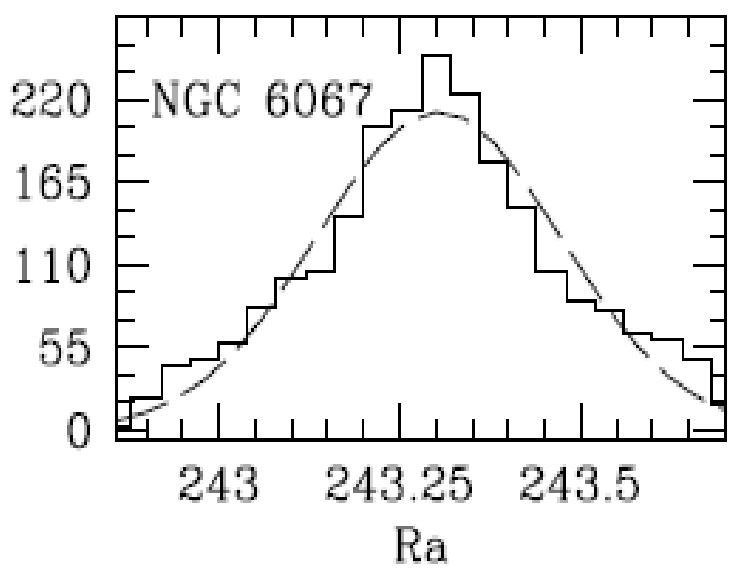

6067 we determined the basic parameters of the cluster. The center of the cluster was estimated as $243.304 \pm 0.01 \mathrm{deg}$ and $-54.225 \pm 0.004 \mathrm{deg}$ in RA and DEC respectively. From the CMD of the cluster we determined the age of the cluster as $129 \pm 03 \mathrm{Myr}$, metallicity as $\mathrm{Z}=0.01$, reddening as $0.37 \pm 0.02 \mathrm{mag}$, distance modulus as $12.1 \pm 0.1 \mathrm{mag}$ and heliocentric distance using distance modulus as $1550.957 \pm$ pc.

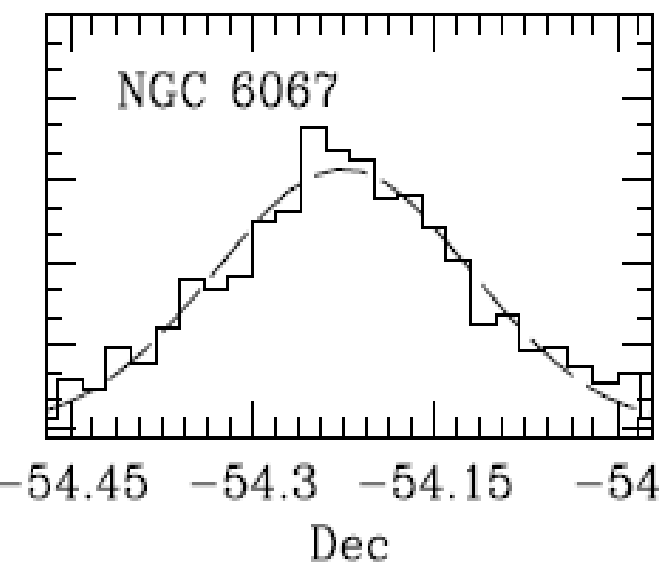

Fig. 1. The histograms of the RA and DEC for the cluster NGC 6067. The dashed curve represent Gaussian fitting.

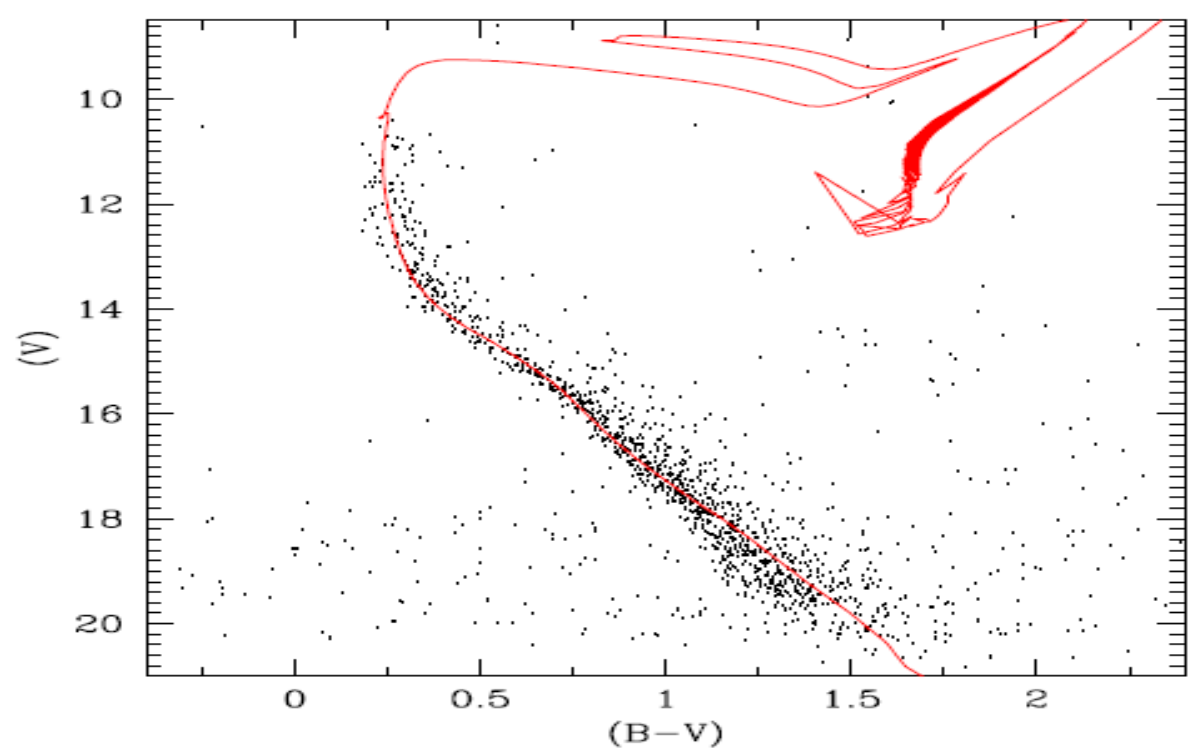

Figure 2 (Color online). The colour-magnitude diagram for the cluster NGC 6067. The isochrone of Marigo et al. (2017) of age $129 \mathrm{Myr}$ and metallicity 0.01 fitted on the main sequence of the cluster. 


\section{Acknowledgements}

We are thankful to the anonymous referee for careful reading of the paper and constructive comments. This analysis has made use of WFI@2.2 and WEBDA database data.

\section{References}

Anderson, J., Bedin, L. R., Piotto, G., Yadav, R. K. S., Bellini, A., 2006, A\&A, 454, 1029.

Durgapal, A. K., Pandey, A. K., 2001, A \& A, 375, 840.

Marigo, P., et al. ,2017, ApJ, 835, 1.

Thackeray, A. D., Wesselink, A. F., Harding, 1962, MNRAS, 124, 445.

Yadav, R. K. S., Sagar, R., 2001, MNRAS, 337, 133 